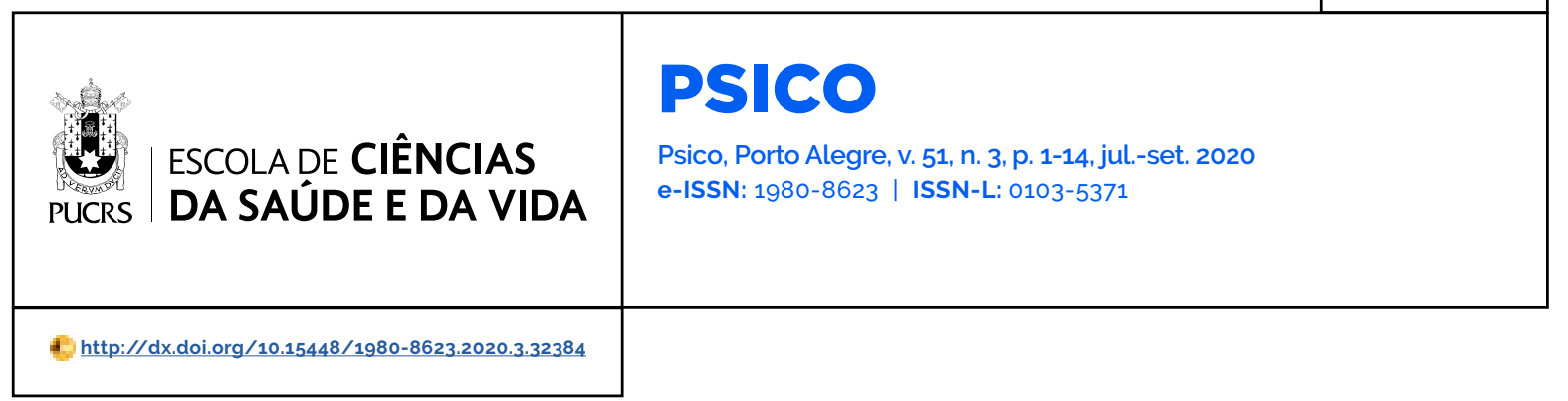

\title{
Revisão sistemática: a Psicologia Positiva e sua aplicação nos contextos do trabalho
}

\author{
Systematic review: positive psychology and its application in the contexts of work \\ Revisión sistemática: psicología positiva y su aplicación en los contextos del trabajo
}

\author{
Adaiane Amélia Baccin ${ }^{1}$ \\ orcid.org/0000-0002-5757-7957 \\ adaiane.baccin@gmail.com
}

\section{Andrea de Fátima Machado da Silva ${ }^{1}$}

orcid.org/0000-0002-9208-3776 deiapsi93@gmail.com

\section{Carlie da Fontoura Taschetto ${ }^{1}$}

orcid.org/0000-0001-8925-7299 carlietaschetto@gmail.com

\section{Jairo Manzoni \\ Rodrigues $^{1}$ \\ orcid.org/0000-0002-1282-3421} jairomanzoni@gmail.com

\section{Priscila Flores Prates ${ }^{\mathbf{1}}$ \\ orcid.org/0000-0002-2340-2286 \\ psico_piti@yahoo.com.br}

\section{Silvio José Lemos Vasconcellos ${ }^{1}$}

orcid.org/0000-0001-6415-7494

silviojlvasco@hotmail.com

Recebido em: 30/10/2018 Aprovado em: 4/4/2020 Publicado em: 21/01/2021.

\section{(c) (i)}

Artigo está licenciado sob forma de uma licença Creative Commons Atribuição 4.0 Internacional.
Resumo: A Psicologia Positiva propõe uma nova abordagem da Psicologia, apresenta o estudo científico das potencialidades humanas e valoriza os aspectos positivos. O presente estudo tem como objetivo investigar a produção científica na área de Psicologia Positiva e sua utilização no contexto do trabalho. O estudo consiste em uma revisão sistemática dos artigos dos últimos 5 anos nas bases de dados Scielo, Pepsic e Lilacs, com os seguintes descritores: "Psicologia Positiva" e "Trabalho" no idioma português, Psicologia Positiva e trabalho no idioma inglês e Psicologia Positiva e trabalho na língua espanhola. De acordo com os critérios de inclusão, 13 artigos foram avaliados nesta revisão. Os resultados mostraram que a maioria dos estudos selecionados abordou a aplicação e o desenvolvimento de escalas que utilizam a Psicologia Positiva; a escala de Engajamento é a mais utilizada, com prevalência de 38\%. Na revisão sistemática, foi possivel confirmar a qualidade das escalas desenvolvidas e validadas. Conclui-se que o uso de constructos de Psicologia Positiva é relevante nos contextos de trabalho. Palavras-chave: psicologia positiva, revisão sistemática, avaliação psicológica

Abstract: The positive psychology proposes a new approach to psychology, presents the scientific study of human potentialities and values personal strengths. The present study aim to investigate scientific production in the area of Positive Psychology and its use in the context of work. The study consists of a systematic review of the articles of the last 5 years in the Scielo. Pepsic and Lilacs databases, with the following descriptors: "Positive Psychology" and "Work" in the Portuguese language, positive psychology and work in the English language and positive psychology and work in the Spanish language. According to the inclusion criteria 13 articles was evaluated in this review. The results showed that the majority of the selected studies approached the application and development of scales that use the positive psychology, the compromise scale is the most used, with a prevalence of $38 \%$. In the systematic review, it was possible to confirm the quality of the scales developed and validated. It is concluded that the use of Positive Psychology constructs is relevant in work contexts.

Keywords: positive psychology, systematic review, psychological assessment

Resumen: La Psicología Positiva propone un nuevo enfoque de la Psicología, presenta el estudio cientíico de las potencialidades humanas y valora las fuerzas personales. El presente estudio tiene como objetivo investigar la producción científica en el área de la Psicología Positiva y su utilización en el contexto del trabajo. El estudio consiste en una revisión sistemática de los artículos de los últimos 5 años en las bases de datos Scielo, Pepsic y Lilacs, con los siguientes descriptores: "Psicologia Positiva" y "Trabajo" en la lengua portuguesa, positive psychology and work en la lengua inglesa y psicologia positiva y trabajo en la lengua española. Atendiendo a los criterios de inclusión, 13 artículos se evaluán en esta revisión. Os resultados mostraron que la mayoria de los estudios seleccionados abordaron la aplicación y lo desarrollo de escalas que utilizan los constructos de la Psicologia Positiva, la escala de compromiso es la más utilizada, con una prevalencia del 38\%. En la revisión sistemática, fue posible confirmar 
la calidad de las escalas desarrolladas y validadas. Se concluye que el uso de los constructos de Psicología Positiva es relevante en los contextos de trabajo.

Palabras clave: psicologia positiva, revisión sistemática, evaluación psicológica

Ao final da década de 1990, surgiu um novo campo de pesquisa na ciência psicológica denominado Psicologia Positiva, que direcionou os estudos aos aspectos saudáveis dos sujeitos (Seligman \& Csikszentmihalyi, 2000). Desde a Segunda Guerra Mundial, o centro das atenções da Psicologia era curar e reparar os danos, abarcando mais os aspectos de saúde e doença. Os referidos autores trouxeram uma nova proposta, a de focar a atenção em aspectos positivos da vida.

Na história da Psicologia, as pesquisas concentravam-se em distúrbios emocionais, sendo restritas à esfera da Psicopatologia. Com o passar dos anos, a Psicologia mostrou-se pouco propositiva no que se refere ao desenvolvimento de uma vida feliz, fazendo com que as forças e as virtudes individuais ficassem em segundo plano ou fossem debatidas sem base científica. Nesses termos, pode-se dizer que a Psicologia Positiva é considerada a ciência que estuda os aspectos positivos da vida humana e suas influências no equilibrio das pessoas (Cogo, 2011). A Psicologia Positiva não desconsidera as dificuldades, mas busca dar enfoque maior nas questões positivas, como qualidades e competências, identificando fatores que promovem qualidade de vida, felicidade e bem-estar, tanto nos individuos quanto em grupos e organizações (Seligman \& Csikszentmihalyi, 2014; Silva \& Boehs, 2017).

A partir desse movimento, a Psicologia vem sendo estudada por diferentes autores na compreensão de sua importância para o desenvolvimento de potencialidades, por meio de um estudo científico de aspectos virtuosos e usuais presentes nos indivíduos (Seligman, 2011). Apesar de serem recorrentes os aspectos que atuam de forma negativa, o interesse nas pesquisas voltadas para o polo positivo tem crescido nas últimas décadas (Rebolo \& Bueno, 2014).

As investigações na área da Psicologia das Organizações e do Trabalho, que antes eram direcionadas à análise dos malefícios físicos e psicológicos à saúde do trabalhador, também foram redirecionadas. Assim, começa a ter destaque a abordagem do comportamento organizacional positivo (Youssef \& Luthans, 2007), fenômenos como o bem-estar laboral, por exemplo, passaram a ocupar cada vez mais a atenção dos pesquisadores organizacionais interessados em aprofundar o conhecimento acerca de suas dimensões, antecedentes e consequentes. Por exemplo, um dos construtos bastante estudado é o engajamento no trabalho, que para a Psicologia Positiva, pode gerar ou aumentar o bem-estar dos individuos no trabalho (Schaufeli et al., 2013).

Da aplicação da Psicologia Positiva ao contexto organizacional, surge o conceito de comportamento organizacional positivo (COP) (positive organizational behavior), iniciado por Luthans (2002). Esse pensador volta-se para o estudo das capacidades psicológicas que têm influência no desempenho organizacional. Ao centrarem a sua atenção apenas nas capacidades que têm impacto no desempenho, os proponentes dessa nova abordagem, pretendem legitimála como uma área de estudo aplicada, que contribui para a gestão mais eficaz das pessoas nas organizações (Palma et al., 2007).

Essa nova e promissora proposta pauta-se, portanto, na ideia de que o cultivo de emoções positivas é possivel para a conquista de mais felicidade. Aplicar os preceitos da Psicologia Positiva no contexto laboral requer que os profissionais da área de gestão de pessoas assumam uma nova postura, buscando visualizar um novo cenário à frente de seus colaboradores. Nesse sentido, utilizar-se das contribuições da área é assimilar novas formas de gerir pessoas, direcionada para o desenvolvimento e o reconhecimento das potencialidades, das forças e das virtudes dos trabalhadores (Cogo, 2011).

Diante desse contexto, a prática da Psicologia Positiva também propõe estimular o desenvolvimento das forças positivas inerentes à pessoa e sugere o investimento em intervenções nos ambientes laborais. Além disso, aborda aspectos relacionados à resiliência e às forças no de- 
senvolvimento humano tais como: sabedoria e conhecimento, coragem, amor, justiça e transcendência (Seligman \& Peterson, 2003). Nessa mesma perspectiva, Cogo (2011) pontua que voltar o foco a intervenções que cultivem a saúde mental reduz o preconceito e o estigma.

No cenário brasileiro, um maior interesse nesse campo data de 1996, quando Hutz, Koller e Bandeira publicaram estudo sobre resiliência e vulnerabilidade. Desde então, foram escritos outros trabalhos voltados para construtos relacionados. É igualmente, portanto, importante destacar que, na atualidade, a Psicologia Positiva encontrase em processo de expansão dentro da ciência psicológica em diversos países, embora possa ser considerado mais recente o fortalecimento dessa abordagem no Brasil (Paludo \& Koller, 2007).

O Instituto Brasileiro de Avaliação Psicológica (IBAP, 2015) demonstra o expressivo crescimento na área desde a criação do Instituto Brasileiro de Avaliação Psicológica e catalogou 46 laboratórios e centros de estudos de Avaliação Psicológica no Brasil, sendo 12 desses localizados na região Sul. Pode-se, nesses termos, citar alguns laboratórios que realizam pesquisa na área da Psicologia Positiva no Brasil, tais como: o Laboratório de Mensuração da Universidade Federal do Rio Grande do Sul (UFRGS), o Laboratório de Psicologia Positiva nas Organizações e no trabalho (LAPPOT) da Universidade Federal de Santa Catarina (UFSC), o Laboratório de Pesquisa em Avaliação Psicológica (LPAP) da UFSC, o Laboratório de Pesquisa em Avaliação Psicológica da Universidade Federal de Ciências da Saúde de Porto Alegre (UFCSPA), o Laboratório de Avaliação Psicológica do Complexo de Ensino Superior de Cachoeirinha (CESUCA), e o Núcleo de Avaliação Psicológica do Delta - NAPSID, da Universidade Federal do Piaui (UFPI). Contamos também com o Grupo de Estudo, Aplicação e Pesquisa em Avaliação Psicológica (GEAPAP) e o Centro de Avaliação Psicológica da UFRGS. E, ainda, o Laboratório de Psicometria e Psicologia Positiva (LP3) da Universidade Federal do Rio de Janeiro (UFRJ), dentre outros que estão contribuindo com o fortalecimento da área no Brasil.
Ressalta-se também que a Psicologia Positiva está voltada para desfazer o viés reducionista e negativo hoje existente em algumas abordagens, com enfoque nas potencialidades e qualidades humanas. Nesse sentido, a resiliência é uma das formas de considerar os fenômenos referentes a uma vida saudável e adaptativa ao longo do desenvolvimento (Yunes, 2003). Para Antunes (2003), a resiliência representa a capacidade de resistência a condições durissimas e persistentes e, dessa forma, dizem respeito à capacidade de pessoas, grupos ou comunidades não só de resistir às adversidades, mas de utilizá-las em seus processos de desenvolvimento pessoal e crescimento social.

Diante das incertezas no contexto atual, as pessoas vivem cada vez mais ameaçadas por realidades externas e internas, Tavares (2002) enfatiza que as sensações de insegurança, ansiedade e angústia conduzem muitos acontecimentos a situações que visam ao desespero. Então a Resiliência é utilizada para aprender a lidar e a conviver, sendo necessário criar e desenvolver defesas físicas e psicológicas. Seligman e Peterson (2003) salientam que para se alcançar êxito em prevenção é necessário e fundamental investir no conhecimento científico sobre as virtudes e as forças pessoais, sendo um aporte da Psicologia Positiva e questões bem presentes no dia a dia de trabalho, o que demonstra a relevância e a atualidade da revisão sistemática proposta.

Diante do exposto, destaca-se que a presente pesquisa tem como objetivo realizar a revisão sistemática por meio da busca e da análise de trabalhos científicos publicados nos últimos cinco anos, a fim de identificar o estado atual de conhecimento produzido sobre o assunto na América Latina. No que se refere aos trabalhos de construção e de validação de instrumentos psicométricos revisados, buscou-se ainda considerar a adequação das amostras, as consistências internas obtidas, bem como as correlações entre as variáveis investigadas e as soluções fatoriais propostas nesses mesmos estudos. 


\section{Método}

A revisão sistemática realizada foi conduzida conforme a metodologia Preferred Reporting Items for Systematic Reviews and MetaAnalyses (PRISMA) e conta com artigos empiricos publicados nos últimos cinco anos, oriundos de buscas em três bases de dados separadamente:Scientific Electronic Library Online (SciELO); Portal de Periódicos Eletrônicos de Psicologia (Pepsic) e Literatura Latino Americana e do Caribe em Ciências da Saúde (Lilacs). Foram utilizados juntos, na mesma busca, os descritores "Psicologia Positiva" no primeiro espaço e "Trabalho" no segundo. Foram efetuadas nas linguas Portuguesa, na inglesa, positive psychology and work; e psicología positiva y trabajo, na lingua Espanhola, abrangendo as pesquisas de toda a América Latina.

Esses descritores foram utilizados como forma de tornar possível identificar a utilização da Psicologia Positiva nos contextos do trabalho. Além disso, não foram considerados os artigos de revisão pelo fato de o interesse pautar-se apenas na utilização da Psicologia Positiva aplicada ou relacionada ao trabalho em diferentes organizações e instituições. As buscas iniciaram no Scielo, depois Pepsic e, por último, no Lilacs, tendo sido desconsiderados manuscritos repetidos, conforme as buscas anteriores.

Na sequência, a Tabela 1 engloba a exposição detalhada da busca dos artigos.

Foram encontrados 92 artigos, desses, 74 foram excluídos após serem aplicados os critérios de elegibilidade. Os motivos das exclusões foram os seguintes para cada estudo: por não versar sobre a Psicologia Positiva, não estar relacionado ao contexto do trabalho, ser estudo bibliográfico e de revisão, por existir repetição nas bases de dados, por não ter sido disponibilizado o estudo por completo. Atendendo aos critérios de inclusão, 18 artigos preencheram os critérios de elegibilidade, e seguindo o método Prisma, desses, dois foram excluídos por se tratar de estudos qualitativos, um por incluir participantes adolescentes, e outros dois foram excluidos considerando um possível viés em cada estudo em decorrência de um número bastante reduzido de participantes. Após a seleção criteriosa dos estudos, treze atenderam aos procedimentos e estão citados na presente revisão sistemática. A análise dos dados dos documentos indicados foi efetuada por meio de uma ficha de leitura, que objetivou identificar quais os conceitos teóricos utilizados, procedimentos metodológicos e os principais resultados dos estudos encontrados. Os critérios utilizados na fase de inclusão foram os seguintes: a) busca de artigos empíricos quantitativos dos últimos cinco anos, de 2015 até 2019; b) artigos publicados na América Latina; e c)artigos relacionados à temática da Psicologia Positiva nos contextos de trabalho. Os novos critérios de exclusão adotados foram: a) artigos de revisão sistemática; b) artigos de outras localidades fora da América Latina; c) artigos com temáticas próximas, mas que não contemplaram o objetivo desta revisão; d) artigos que estavam repetidos em mais de uma das bases de dados; e) artigos qualitativos; f) artigos com participantes adolescentes que não estão trabalhando; e g) artigos com amostra muito pequena de participantes, indicando possivel viés nas análises.

TABELA 1 - Resumo dos resultados da busca

\begin{tabular}{|c|c|c|c|c|c|}
\hline $\begin{array}{l}\text { Bases de } \\
\text { dados }\end{array}$ & $\begin{array}{l}\text { Artigos } \\
\text { resgatados } \\
\text { pela busca }\end{array}$ & $\begin{array}{l}\text { Artigos } \\
\text { excluidos }\end{array}$ & $\begin{array}{l}\text { Artigos } \\
\text { analisados }\end{array}$ & $\begin{array}{l}\text { Excluídos } \\
\text { após análise } \\
\text { temática }\end{array}$ & $\begin{array}{l}\text { Incluídos no } \\
\text { artigo }\end{array}$ \\
\hline SCIELO & 16 & 6 & 10 & O & 10 \\
\hline LILACS & 65 & 42 & 23 & 19 & 4 \\
\hline PEPSIC & 11 & 4 & 7 & 2 & 5 \\
\hline
\end{tabular}

Fonte: Elaborada pelos autores. 
Inicialmente, foram inseridos os descritores citados e protocolados todos os artigos encontrados em uma planilha Excel na qual foram sendo armazenadas as informações como título, ano de publicação, objetivo, resultados, conclusão, autores, data da busca dos artigos e endereços eletrônicos para facilitar a localização do artigo, bem como para inserir nas referências bibliográficas, para análise e apresentação dos dados. Posteriormente, foram aplicados os filtros de acordo com os critérios de elegibilidade e efetuadas as leituras de todos os artigos para a identificação do atendimento aos critérios. $\mathrm{Na}$ Figura 1, apresentamos o fluxograma da busca.

Figura 1 - Fluxograma da busca

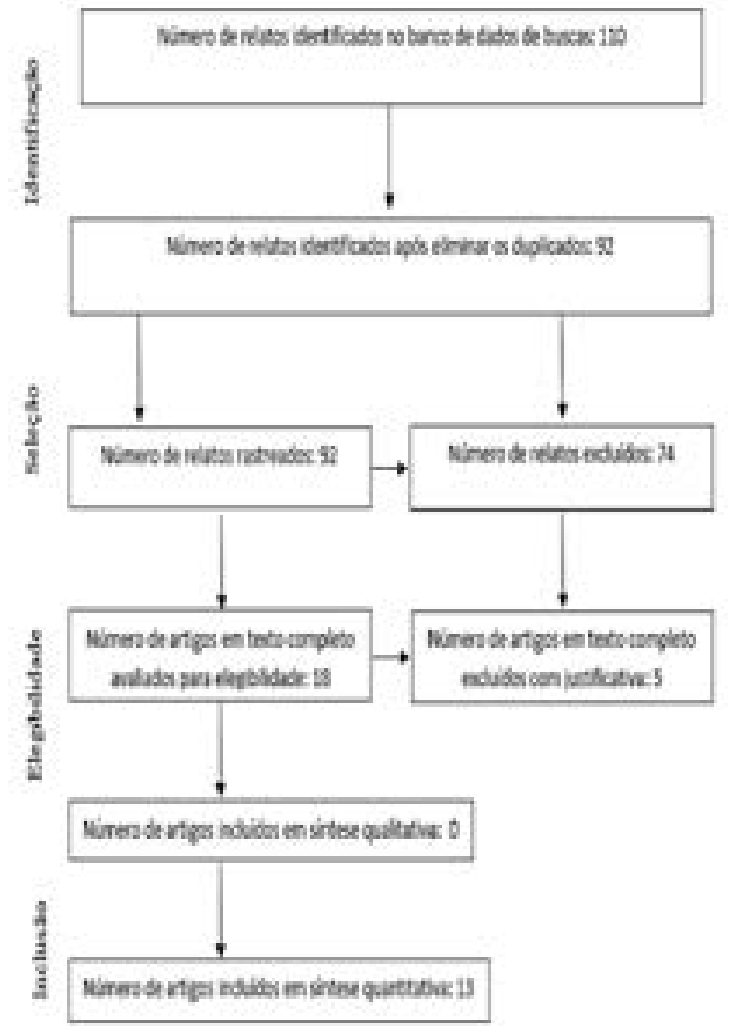

Fonte: Elaborada pelos autores.

No que se refere à justificativa para a exclusão de artigos completos, na fase de seleção dos estudos foi excluído o artigo de Bonifácio e Scorsolini-Comin (2019) por se tratar de um relato de uma intervenção clínica na abordagem da Psicologia Positiva e o de Bastianello e Hutz (2015) por ser estudo qualitativo e apresentação de abordagens teóricas. Também foi excluído o artigo de Campos e Noronha (2016) por envolver adolescentes, pois ainda não se encontram no contexto laboral, alvo da presente revisão. Ainda atendendo aos critérios de seleção dos estudos no método Prisma, os artigos dos autores Antoniolli et al. (2017) e de Dalanhol et al. (2017) com 16 e 82 participantes respectivamente. Ambos foram excluídos devido ao risco de viés dos estudos por serem amostras pequenas.

\section{Resultados}

Após a realização das buscas e análise dos artigos, elaboramos a Tabela 2, que apresenta os resultados encontrados. Consta na tabela o título, ano de publicação, objetivos, resultados encontrados, conclusão e referências.

Entre os quatro estudos publicados em 2015, três deles trataram da elaboração e da validação de escalas utilizando os constructos da Psicologia Positiva. Tais como o estudo 1, com 1167 participantes, no qual foram realizadas a validade de conteúdo e de construto com os 17 itens da escala, e nove da versão reduzida, em que foram feitas as análises fatoriais exploratória e confirmatória. Outro estudo foi 02 , também com 1167 participantes, no qual foi verificada a consistência interna de 0.95 e as correlações entre os fatores foram de 0,81 a 0,82. E o estudo 3. com 491 participantes, no qual também foram efetuadas as análises confirmatórias que revelaram apresentar bons indices de consistência interna. Em relação à correlação dos escores da escala de engajamento com variáveis externas, verificou-se que a escala apresentou correlações fortes e positivas com os escores de florescimento no trabalho, de satisfação no trabalho e de paixão harmoniosa pelo trabalho (Vasquez et al., 2015: Chinelato et al., 2015).

O estudo 4 de Silva Filho e Ferreira (2015) com 344 participantes, no qual foram realizadas a análise fatorial exploratória e estatística descritiva e verificado o coeficiente de correlação, as análises de regressão múltipla evidenciaram que as dimensões da espiritualidade no trabalho associadas ao sentido do trabalho e 
TABELA 2 - Psicologia Positiva nas Instituições/Organizações: artigos encontrados

\begin{tabular}{|c|c|c|c|c|}
\hline $\begin{array}{l}\text { No-Título do } \\
\text { artigo }\end{array}$ & Referência & Objetivos & Resultados & Conclusão \\
\hline $\begin{array}{l}\text { 1. Adaptation and } \\
\text { Validation of the } \\
\text { Brazilian Version of } \\
\text { the Utrecht Work } \\
\text { Engagement Scale. }\end{array}$ & $\begin{array}{l}\text { Vazquez et al. } \\
\text { (2015) }\end{array}$ & $\begin{array}{l}\text { Adaptar e obter evi- } \\
\text { dências de validade } \\
\text { da versão brasileira da } \\
\text { Utrecht Work Engage- } \\
\text { ment Scale (UWES). }\end{array}$ & $\begin{array}{l}\text { Análises fatoriais explora- } \\
\text { tória e confirmatória de- } \\
\text { monstraram que a melhor } \\
\text { solução seria unifatorial. } \\
\text { A consistência interna foi } \\
\text { elevada. Foram encon- } \\
\text { tradas diferenças de } \\
\text { engajamento em relação } \\
\text { à idade. }\end{array}$ & $\begin{array}{l}\text { Os resultados indicam } \\
\text { que a versão brasileira da } \\
\text { UWES é válida e reforçam } \\
\text { a ideia de que } \\
\text { o engajamento no } \\
\text { trabalho é um construto } \\
\text { universal. Porém, algumas } \\
\text { diferenças culturais foram } \\
\text { detectadas e são discuti- } \\
\text { das no presente trabalho. }\end{array}$ \\
\hline $\begin{array}{l}\text { 2. Normatização da } \\
\text { versão Brasileira } \\
\text { da Escala Utrecht } \\
\text { de Engajamento no } \\
\text { Trabalho. }\end{array}$ & $\begin{array}{l}\text { Magnan et al. } \\
\text { (2015) }\end{array}$ & $\begin{array}{l}\text { Apresentar as normas } \\
\text { para interpretação dos } \\
\text { resultados obtidos pela } \\
\text { aplicação da versão } \\
\text { adaptada e validada } \\
\text { para o Brasil da UWES. }\end{array}$ & $\begin{array}{l}\text { A amostra do presente } \\
\text { estudo apresentou escore } \\
\text { médio no engajamento de } \\
4,4 \% \text { (DP=1,2). O grupo com } \\
\text { maior idade apresentou } \\
\text { média de engajamento } \\
\text { (M=4,8; DP=1,1) mais eleva- } \\
\text { da que o grupo em início } \\
\text { da vida laboral }(M=4,2, \\
\text { DP=1,2) e em formação } \\
\text { profissional }(M=4,4 ; D P=1,0)\end{array}$ & $\begin{array}{l}\text { Os resultados encontra- } \\
\text { dos corroboram estu- } \\
\text { dos internacionais que } \\
\text { sugerem diferenças nas } \\
\text { médias de engajamento } \\
\text { quando se considera o } \\
\text { grupo ocupacional. }\end{array}$ \\
\hline $\begin{array}{l}\text { 3. Evidence of } \\
\text { Validity of the Job } \\
\text { Crafting Behaviors } \\
\text { Scale. }\end{array}$ & $\begin{array}{l}\text { Chinelato et al. } \\
\text { (2015) }\end{array}$ & $\begin{array}{l}\text { Adaptar e reunir evidên- } \\
\text { cias de validade da } \\
\text { Escala de Comporta- } \\
\text { mentos de Redesenho } \\
\text { do Trabalho para o } \\
\text { contexto brasileiro. }\end{array}$ & $\begin{array}{l}\text { As análises fatoriais confir- } \\
\text { matórias revelaram que o } \\
\text { instrumento final se com- } \\
\text { pôs de três dimensões } \\
\text { que apresentaram bons } \\
\text { indices de consistência in- } \\
\text { terna e correlações positi- } \\
\text { vas moderadas ou baixas } \\
\text { com o engajamento no } \\
\text { trabalho, o capital psico- } \\
\text { lógico positivo, os afetos } \\
\text { positivos no trabalho e o } \\
\text { desempenho intrapapéis } \\
\text { de trabalho. }\end{array}$ & $\begin{array}{l}\text { A escala demonstrou } \\
\text { evidências de validade, o } \\
\text { que recomenda seu uso } \\
\text { para futuras pesquisas } \\
\text { sobre as mudanças que } \\
\text { os individuos realizam em } \\
\text { seu trabalho. }\end{array}$ \\
\hline $\begin{array}{l}\text { 4. O impacto da } \\
\text { espiritualidade no } \\
\text { trabalho sobre o } \\
\text { bem-estar laboral. }\end{array}$ & $\begin{array}{l}\text { Silva Filho et al. } \\
(2015)\end{array}$ & $\begin{array}{l}\text { Investigar o poder pre- } \\
\text { ditivo da espiritualidade } \\
\text { no trabalho sobre o } \\
\text { bem-estar laboral. }\end{array}$ & $\begin{array}{l}\text { As análises de } \\
\text { regressão múltipla evi- } \\
\text { denciaram que as dimen- } \\
\text { sões da espiritualidade } \\
\text { no trabalho associadas ao } \\
\text { sentido e ao sentimento } \\
\text { de comunidade predis- } \\
\text { seram positiva e signifi- } \\
\text { cativamente a satisfação, } \\
\text { o comprometimento } \\
\text { organizacional afetivo e os } \\
\text { afetos positivos dirigidos } \\
\text { ao trabalho. }\end{array}$ & $\begin{array}{l}\text { Tais resultados permiti- } \\
\text { ram a conclusão de que, } \\
\text { quando as necessidades } \\
\text { espirituais dos membros } \\
\text { da organização são satis- } \\
\text { feitas, eles desenvolvem } \\
\text { maior ligação afetiva com } \\
\text { a organização. }\end{array}$ \\
\hline
\end{tabular}




\section{No-Título do artigo \\ Referência Objetivos Resultados}

(Oliveira \& Fer- Investigar o impacto

reira, 2016)

percepções de

justiça organizacio-

nal e da resiliência

sobre o engajamen-

to no trabalho.

6. Evidências adi-

cionais de validade

da UWES-9 em

amostras brasileiras.
Ferreira et al.

(2016)

(1)

tiça organizacional e da

resiliência sobre o en-

gajamento no trabalho.

mediante um estudo

de levantamento de

natureza transversal.

Reunir evidências de

validade da versão

reduzida da escala

(UWES-9) por meio de

trabalho
7. Evidências

de validade da Es-

cala de Enriqueci-

mento TrabalhoFa-

milia em amostras

brasileiras.

\section{das percepções de jus-}

análises de estrutura

interna, de invariância

por gênero e setor de

trabalho e de relações

com variáveis externas.

\section{Gabardo-Martins Adaptar e buscar}

et al. (2016)

evidências de validade

da Escala de Enriqueci-

mento TrabalhoFamília

no contexto brasileiro.

Gottardo et al.

(2016)

avaliações autorre-

ferentes e bem-es-

tar de profissionais

de saúde.
A análise de regressão

múltipla evidenciou que

as percepções de justiça

distributiva e interpessoal,

bem como a resiliência

predisseram positiva-

mente o engajamento no

Os modelos indicaram

que a estrutura uni-

dimensional obteve

melhor ajuste e que os

parâmetros dos itens não

foram significativamente

enviesados pelo sexo,

setor e tempo de trabalho.

Tem correlações positivas

fortes com sentimentos

positivos associados ao

trabalho.

O fator geral apresentou

um Alfa de Cronbach igual

a 0.94. A subescala de En-

riquecimento do Trabalho

para a Familia apresentou

uma correlação positiva

elevada $(r=0,73 ; p<0,01)$

com a Escala de Satisfa-

ção no Trabalho

As análises de regressão

múltipla hierárquica reve-

laram que quanto maior

a percepção de suporte

social informacional de

colegas ou supervisores

no trabalho, maiores os

índices de satisfação no

trabalho e de afetos diri-

gidos ao trabalho; quanto

maiores as avaliações

autorreferentes, maiores

os indices de satisfação

no trabalho e de afetos

dirigidos ao trabalho.
Concluiu-se que a justiça

organizacional e a resili-

ência consistem recur-

sos motivacionais que

promovem o engajamento

no trabalho.

As propriedades psicométricas do instrumento recomendam seu uso

futuro em investigações destinadas a avaliar o engajamento no trabalho em amostras brasileiras.

Concluiu-se que a escala

é um instrumento com

evidências de validade adequadas, o que possibilita seu uso em situações futuras de pesquisa da interface trabalho-familia.

Concluiu-se que o suporte informacional é de especial relevância para o bem-estar dos profissionais de saúde. 


\section{No-Título do artigo \\ Referência Objetivos \\ Resultados}

Propor um instrumento para a mensuração da interface positiva entre trabalho e familia.
Aguiar et al.

9. Interface positiva trabalho e familia: proposta de instrumento e evidências de validade.
(2018)
Os resultados apontaram o modelo com dois fatores como o que melhor representa a dimensionalidade do construto. A versão final da medida foi composta por 19 itens: 9 (interface positiva trabalho-familia) e 10 à (interface positiva familia-trabalho).

\section{Inventário de} Flow no Trabalho: evidências de validade da estrutura interna e critério.

11. Anteceden-tes do engajamento no trabalho de professores do ensino superior no Brasil.

12. Satisfação

Ocupacional de Médicos: O Impacto de Demandas e Recursos.

$\begin{array}{ll}\text { Salessi et al. } & \text { Adaptar e validar o } \\ \text { (2018) } & \text { Inventário de Flow no } \\ & \text { Trabalho de Bakker na } \\ & \text { Argentina. }\end{array}$

Análises de equações estruturais exploratórias indicaram a adequação de um modelo tri-fatorial com 10 itens. As correlações positivas com paixão, satisfação e implicação no trabalho indicaram ainda apropriada validade.

Ercali et al. Identificar e analisar os (2019) principais antecedentes, as demandas e os recursos externos de trabalho que estão relacionados com o engajamento na esfera laboral dos docentes de ensino superior brasileiros.

Pereira et al. Identificar o impacto de (2019) um recurso do trabalho (enriquecimento trabatho-familia) e de uma demanda (sobrecarga) sobre a satisfação laboral dos médicos, bem como o papel mediador de um recurso pessoal (paixão harmoniosa pelo trabalho) nessas relações
Os achados mostram que os docentes possuem escores medianos de engajamento, porém muito próximos de um saúde mental deles. As demandas qualitativas e os recursos execução de tarefas são os mais expressivos na vida laboral desses profissionais.

Os resultados dos efeitos diretos indicaram que todos eram significativos (enriquecimento da familia e satisfação ocupacional: Beta=0,48; $p<0,001$; sobrecarga e satisfação ocupacional: Beta=-0,15; $p<0,01$; paixão harmoniosa e satisfação ocupacional: Beta $=0,35 ; p<0,01$ ) limite que pode afetar a
Acredita-se que a medida proposta poderá contribuir com a perspectiva da Psicologia Positiva nas organizações.

\section{Esses resultados mostram que a versão argentina do instrumento possui pro- priedades psicométricas adequadas.}

Em geral, os docentes de instituições privadas percebem as demandas e os recursos de trabalho de maneira mais favorável que os que atuam em instituições públicas.

A identificação e o fortalecimento do sentimento de paixão pelo trabalho dos médicos podem contribuir para a melhoria de seu bem-estar laboral e, em consequência, o de seus familiares e clientes. 


\begin{tabular}{|c|c|c|c|c|}
\hline $\begin{array}{l}\text { No-Título do } \\
\text { artigo }\end{array}$ & Referência & Objetivos & Resultados & Conclusão \\
\hline $\begin{array}{l}\text { 13. Evidências ini- } \\
\text { ciais de validade da } \\
\text { escala de liderança } \\
\text { virtuosa. }\end{array}$ & $\begin{array}{l}\text { Guimarães et al. } \\
\text { (2019) }\end{array}$ & $\begin{array}{l}\text { Reunir evidências } \\
\text { iniciais de validade no } \\
\text { contexto brasileiro para } \\
\text { a Escala de Liderança } \\
\text { Virtuosa. }\end{array}$ & $\begin{array}{l}\text { Os fatores da escala } \\
\text { de Liderança Virtuosa } \\
\text { apresentaram correlações } \\
\text { positivas altas com todos } \\
\text { os fatores da Escala de } \\
\text { Liderança Autêntica, com } \\
\text { os resultados dessas } \\
\text { correlações variando de } \\
\text { r=0,80; p<0,01 a r=0,93: } \\
\text { p<0,01. Os fatores da Es- } \\
\text { cala de Liderança Virtuosa } \\
\text { também apresentaram } \\
\text { correlações positivas altas } \\
\text { com a Escala de Satisfa- } \\
\text { ção no Trabalho ( } r=0,54 ; \\
\text { p<0,01 a r=0,59; } p<0,01 \text { ). }\end{array}$ & $\begin{array}{l}\text { A Escala de Liderança } \\
\text { Virtuosa apresentou boas } \\
\text { qualidades psicomé- } \\
\text { tricas em uma amostra } \\
\text { brasileira. }\end{array}$ \\
\hline
\end{tabular}

Fonte: Elaborada pelos autores.

ao sentimento de comunidade mostraram-se preditivas em relação à satisfação no trabalho, ao comprometimento organizacional afetivo e aos afetos positivos relacionados ao trabalho.

Entre os quatro estudos publicados no ano de 2016, os estudos 6 e 7 tiveram como foco as escalas com a investigação dos constructos justiça organizacional, resiliência, engajamento no trabalho, enriquecimento trabalho familia e bem-estar, as quais buscaram efetuar correlações e investigar evidências de validade de estrutura interna. Em ambos os estudos se verificou que todos os fatores revelaram boa consistência interna e foram devidamente explicados ao longo dos artigos no que se refere às soluções fatoriais encontradas. $O$ estudo 6 aprofundou as análises dos participantes pois apresentou outros indicadores de validade a partir da invariância por gênero, setor público ou privado e tempo de trabalho - não apresentadas no estudo 7, que não fez tais diferenciações. Os dados obtidos no estudo 7 demonstraram que a subescala de Enriquecimento do Trabalho para a Familia apresentou uma correlação positiva elevada com a Escala de Satisfação no Trabalho e com a Escala de Satisfação com a Vida e sinalizou que quando a vida em familia leva os individuos a auferirem maior qualidade de vida em seu trabalho, mais satisfeitas as pessoas se sentem com a sua vida em geral. Os instrumentos evidenciaram validade adequada, o que possibilita seu uso em situações futuras de pesquisas (Ferreira et al., 2016; Gabardo-Martins et al., 2016).

Em outros dois estudos publicados em 2016. sendo eles ( 8 e 5) com amostra de 302 e 435 participantes, respectivamente, foram verificadas as correlações ente as variáveis e feitas as análises de regressão. No estudo 8 , o resultado demonstra que à medida que aumenta a percepção de suporte social informacional no trabalho, aumentam os indices de satisfação no trabalho e de afetos dirigidos ao trabalho, com relevância para o bem-estar dos trabalhadores. Já no estudo 5. as análises de regressão múltipla predisseram positivamente. Os dados obtidos evidenciaram que, dentre as percepções das quatro dimensões de justiça organizacional, apenas as de justiça distributiva e de justiça interpessoal constituíram-se preditoras positivas e significativas do engajamento no trabalho, sendo que a primeira se destacou como a principal preditora da variável critério. Ambas as temáticas dos estudos são de relevância para o bem-estar dos profissionais e consistem em recursos motivacionais que promovem o engajamento no trabalho (Gotardo \& Ferreira, 2015; Oliveira \& Ferreira, 2016). 


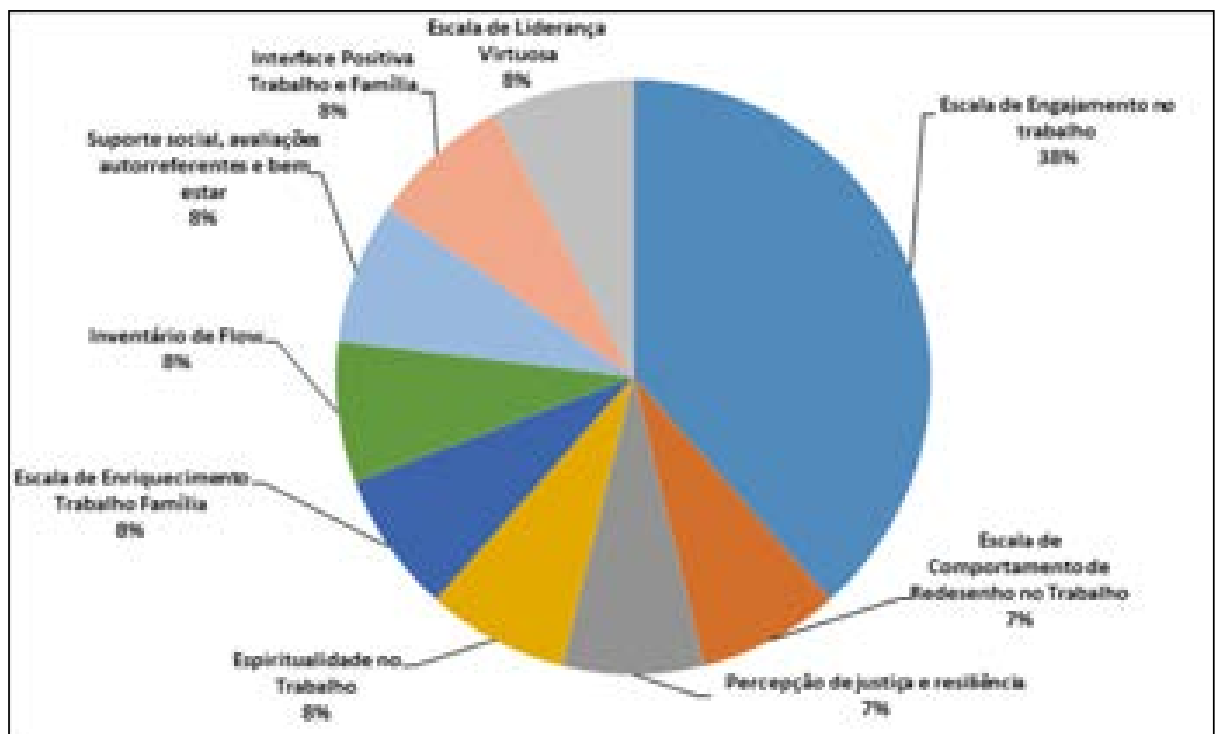

Figura 2 - Constructos teóricos identificados na revisão sistemática Fonte: Elaborada pelos autores.

A prevalência de estudos envolvendo escalas com os constructos da Psicologia Positiva se destacou em 2018, com o artigo 9, com 446 participantes, que teve como objetivo propor um instrumento para a mensuração da interface positiva entre trabalho e familia. Para isso, foi efetuada uma análise fatorial exploratória e foram analisados os indices de consistência interna da medida por meio de alfa de Cronbach que demonstraram evidências de validade. Já no estudo 10, com 442 participantes, que objetivou adaptar e validar o Inventário de Flow no Trabalho, foram realizadas as análises exploratórias, verificadas as correlações e analisadas outras propriedades psicométricas. A pesquisa demonstrou possuir propriedades psicométricas adequadas e os modelos propostos podem contribuir com a concepção da pertinência da Psicologia Positiva nas organizações (Aguiar \& Bastos, 2018; Salessi \& Omar, 2018).

Dentre os estudos recentes, três datam de 2019 e envolvem a utilização de escalas de avaliação, abrangem engajamento no trabalho. O estudo 11, com 506 participantes, no qual foi feita estatística descritiva e análise de variância, obteve alfa de Cronbach de 0,94 demonstrando boa confiabilidade interna; o estudo 12, com 284 participantes, teve boa consistência interna; e no estudo 13, que contou com 417 participantes, os fatores da escala de Liderança Virtuosa, no estudo 13, apresentaram correlações positivas altas com todos os fatores da Escala de Liderança Autêntica, com os resultados dessas correlações variando de $r=0,80 ; p<0,01$ a $r=0,93 ; p<0,01$. Os fatores da Escala de Liderança Virtuosa também apresentaram correlações positivas altas com a Escala de Satisfação no Trabalho $(r=0,54 ; p<0,01$ a r $=0.59 ; p<0,01)$ demonstrando boas qualidades psicométricas (Mercali \& Costa, 2019; Pereira et al., 2019; Guimarães et al., 2019).

Para a seleção dos estudos desta revisão, também foi considerado o tamanho da amostra, sendo selecionados os artigos com amostras de 284 até 2.897 participantes, reduzindo assim o viés dos estudos, além da certificação de que foi realizada a verificação das correlações, os indices de consistência interna e efetuadas as análises das propriedades psicométricas necessárias em cada estudo. Dessa forma, podemos destacar os elevados indices de confiabilidade e a indicação da utilização das escalas, bem como a aplicação dos construtos da Psicologia Positiva nos ambientes laborais. No Brasil, a construção e a validação de escalas estão em expansão e nesta revisão sistemática foi possivel confirmar a qualidade das escalas que têm sido desenvolvidas e validadas no Brasil (Instituto Brasileiro de Avaliação Psicológica, 2015). 
Na Figura 2, acima, o gráfico demonstrativo dos construtos encontrados nas pesquisas.

\section{Discussão}

O presente estudo buscou apresentar as formas de utilização da Psicologia Positiva nos contextos de trabalho. Para tanto, foram analisados 18 estudos triados a partir dos critérios de inclusão e exclusão descritos. Considerase que os resultados possibilitaram responder de forma satisfatória aos objetivos propostos, propiciando a descrição de aspectos relevantes das pesquisas analisadas.

No que diz respeito às caracteristicas metodológicas apresentadas nos artigos, observa-se a prevalência de produções científicas que envolvem a elaboração, normatização e utilização de instrumentos de avaliação acerca dos constructos da Psicologia Positiva. A análise dos delineamentos dos estudos permitiu identificar que as pesquisas apresentaram trabalhos de construção e de validação de instrumentos psicométricos com boa adequação das amostras. Também promoveram boa investigação sobre os constructos em estudo, os quais corroboram com as afirmações de Silva Filho e Ferreira (2015) que consideram temáticas relevantes para serem desenvolvidas em atividades que se relacionem diretamente com a aplicação no contexto de trabalho (Silva Filho \& Ferreira, 2015).

A presente revisão sistemática mostrou que a maioria dos artigos encontrados utiliza a Psicologia Positiva como paradigma de trabalho, sendo poucas as propostas de intervenção. Os estudos efetuados na perspectiva da Psicologia Positiva procuram discutir, entender e promover questões que se relacionam à saúde e aos fatores positivos do ser humano, aspectos que contribuem com o desenvolvimento do comportamento organizacional positivo (Luthans, 2002). As intervenções em Psicologia Positiva, visam o aumento das emoções positivas e o bem-estar dos participantes, por meio da indução de atividades e de exercícios (Seligman, 2011). Também podem ser realizados os denominados programas de desenvolvimento positivo, que são intervenções com maior amplitude, com foco em construir qualidades positivas nas pessoas e em promover aspectos positivos do desenvolvimento. Tais abordagens podem ser ampliadas em estudos futuros no contexto nacional.

Por intermédio da pesquisa proposta. também pode ser observado que entre as 13 publicações que utilizaram a Psicologia Positiva e seus constructos aplicados nos contextos que envolvem o trabalho nas mais variadas áreas, instituições ou organizações, no que diz respeito às escalas utilizadas nas pesquisas, destaca-se a Escala de Engajamento no Trabalho, a qual foi utilizada em diferentes estudos.

As pesquisas que envolvem o constructo da Psicologia Positiva, denominado engajamento no trabalho, correspondem a 38\% dos casos ( 1,2 , $5,6,11$ ), ou seja, cinco dos 13 estudos utilizam o engajamento para as pesquisas e a construção de escalas. Verificou-se, ainda, que a Escala de Engajamento no Trabalho (UWES-9) de Vazquez et al. (2015) foi a mais utilizada. A citada escala de engajamento apontou correlação positiva com sentimentos positivos associados ao trabalho. De acordo com Ferreira et al. (2016), o instrumento demonstrou em todos os estudos ter propriedades psicométricas adequadas para ser utilizado, o que pode ser expressivo pelo fato de que o tema engajamento é um dos temas centrais da Psicologia Positiva (Seligman, 2011).

As demais escalas utilizadas foram: Escala de Percepção de Suporte Social no Trabalho: Escala de Avaliações Autorreferentes; Escala de Satisfação Geral no Trabalho; Escala de Afetos Positivos no Trabalho; Escala de Espiritualidade no Trabalho; Escala de Comprometimento Organizacional Afetivo; Escala de Interação Trabalho Familia; Escala de Enriquecimento Trabalho Familia; Escala de Valores Relativos ao Trabalho: Escala de Autoeficácia Geral; e Escala de Bem-estar Subjetivo. Percebe-se. assim, a variedade de escalas que tem como base constructos da Psicologia Positiva e são de grande relevância para a realização de pesquisas nesse campo.

No que se refere aos fenômenos em estudo, 
observou-se investimento nas pesquisas que envolvem a avaliação da qualidade de vida, a identificação das forças e das virtudes, o bemestar psicológico, o engajamento no trabalho, o otimismo e o suporte social, além do nível de bem-estar no trabalho e a justiça organizacional, e, ainda, o levantamento das dificuldades da decisão profissional e a avaliação do otimismo e dos fatores psicossociais associados.

\section{Considerações finais}

Este artigo possibilitou a compreensão do panorama da Psicologia Positiva no cenário científico, permitindo refletir sobre aspectos pouco explorados pela produção científica, com caracteristicas promissoras. Considerando a própria abrangência da proposta, capaz de abarcar outros paises da América Latina, entendese também que algumas dessas inferências também podem ser estendidas para paises próximos, sendo que a cooperação internacional em termos científicos pode ainda ser aprimorada nessa área.

Um dos possiveis limitadores deste estudo pode ter sido a utilização de apenas dois descritores "psicologia positiva" e "trabalho". Um fato que restringiu o foco da revisão a estudos específicos na área da Psicologia e aplicados ao trabalho. Sugere-se para futuros estudos a realização de uma análise de estudos e discussões realizadas por outras áreas, através da utilização de outros descritores. Além disso, é importante ressaltar ainda que a Psicologia Positiva se propõe a ir além dos construtos teóricos e campos de atuação contemplados neste artigo, cuja delimitação, entretanto, mostrou-se necessária.

\section{Referências}

Aguiar, C. V. N. \& Bastos, A. V. B. (2018). Interfaces entre o trabalho e a familia: questões conceituais e empíricas. Estudos de Psicologia, 23(3), 212-223. http://dx.doi.org/10.22491/1678-4669.20180021
Antoniolli, L., Echevarria-Guanilo, M. E., Martins, C. L." Amestoy, S. C., Longaray, T. M., \& de-Souza, S. B. C. (2017). Coping e estresse na equipe de enfermagem de um centro de tratamento de queimados Revista brasileira de queimaduras, 16(3), 174-180. Recuperado de http://Www.rbqueimaduras.com.br/ details/390/pt-BR/coping-e-estresse-na-equipede-enfermagem-de-um-centro-de-tratamento-dequeimados

Antunes, C. (2003). Resiliência: a construção de uma nova pedagogia para uma escola pública de qualidade. Rio de Janeiro: Vozes.

Bastianello, M. R. \& Hutz, C. S. (2015). Do otimismo explicativo ao disposicional: a perspectiva da psicologia positiva. Psico-USF, 20(2), 237-247. https:// doi.org/10.1590/1413-82712015200205

Bonifácio, D. P. \& Scorsolini-Comin, Fabio. (2019) Preparação para a aposentadoria: relato de uma intervenção clínica na abordagem da psicologia positiva. Revista da Abordagem Gestáltica, 25(3), 237245. https://dx.doi.org/10.18065/RAG.2019v25n3.2

Campos, R. R. F. \& Noronha, A. P. P. (2016). A relação entre indecisão profissional e otimismo disposicional em adolescentes. Temas em Psicologia, 24(1), 219232. https://doi.org/10.9788/TP2016.1-15

Chinelato, R. S. C., Ferreira, M. C., \& Valentini, F. (2015). Evidence of validity of the job crafting behaviors scale. Paidéia, 25(62), 325-332. https://dx.doi. org/10.1590/1982-43272562201506

Cogo, P. S. F. (2011). Psicologia positiva, uma nova ciência do comportamento humano no trabalho. Negócios e Talentos, 8(1), 1527. Recuperado de https://seer.uniritter. edu.br/index.php?journal=negocios\& page $=$ article $\& \mathrm{p}=\mathrm{view} \&$ path $\% 5 \mathrm{~B} \% 5 \mathrm{D}=642$

Dalanhol, N. S., Freitas, C. P. P., Machado, W. L. Hutz, C. S., \& Vazquez, A. C. S. (2017). Engajamento no trabalho, saúde mental e personalidade em oficiais de justiça. Psico, 48(2), 109-119. http://dx.doi. org/10.15448/1980-8623.2017.2.25885

Ferreira, M. C., Valentini, F., Damásio, B. F., Mourão, L., Porto, J. B., Chinelato, R. S. Novaes, V. P., \& Pereira, M. M. (2016). Evidências adicionais de validade da UWES-9 em amostras brasileiras Estudos de Psicologia, 21(4), 435-445. https://dx.doi. org/10.5935/1678-4669.20160042

GabardoMartins, L. M. D., Ferreira, M.C., \& Valentini, F. (2016). Evidências de validade da escala de enriquecimento trabalhofamilia em amostras brasileiras. Revista Psicologia: Teoria e Prática, 18(1), 100112. https://doi.org/10.15348/1980-6906

Gotardo, L. F. S. \& Ferreira, M. C. (2015). Suporte social, avaliações autorreferentes e bem-estar de profissionais de saúde. Arquivos Brasileiros de Psicologia, 67(1), 146-160. Recuperado de http://pepsic.bvsalud.org/scielo.php? script=sci_ arttext\&pid=S1809-52672015000100011 
Guimarães, M., Ferreira, M. C., \& Pereira, M. (2019). Evidências iniciais de validade da escala de liderança virtuosa. Estudos de Psicologia, 36, 01-10. https:// dx.doi.org/10.1590/1982-0275201936e170101

Hutz, C. S. (2014). As origens da psicologia positiva e os primeiros estudos brasileiros. Avaliação em Psicologia Positiva. Porto Alegre: Artes Médicas.

Hutz, C. S., Koller, S. H., \& Bandeira, D. R. (1996). Resiliência e vulnerabilidade em crianças em situação de risco. In S. H. Koller (Org.), Aplicações da psicologia na melhoria da qualidade de vida (Coletâneas da Anpepp, v. 1, n. 12, pp. 79-86). Porto Alegre: Associação Nacional de Pesquisa e Pós-graduação em Psicologia. Recuperado de https://Www.anpepp. org.br/acervo/Colets/v1n12ao6.pdf

Instituto Brasileiro de Avaliação Psicológica. (2015). Catálogo dos laboratórios de avaliação psicológica no Brasil. Recuperado de http://Www.ibapnet.org.br/ congress02015/arquivos/catalogo_dos_laboratorios_. de_avaliacao_psicologica_no_brasil.pdf

Luthans, F. (2002). Positive organizational behavior: developing and managing psychological strengths. Academy of Management Executive. 16(1). 57-72. https://doi.org/10.5465/ame.2002.6640181

Mercali, G. D. \& Costa, S. G. (2019). Antecedentes do engajamento no trabalho dos docentes de ensino superior no Brasil. Revista de Administração Mackenzie, 20(1), 01-28. https://dx.doi. org/10.1590/1678-6971/eramg190081

Oliveira, D. F. \& Ferreira, M. C. (2016). O impacto das percepções de justiça organizacional e da resiliência sobre o engajamento no trabalho. Estudos de Psicologia, 33(4), 747-755. https://doi. org/10.1590/1982-02752016000400017

Palma, P. J., Cunha, M. P., \& Lopes, M. P. (2007). Comportamento organizacional positivo e empreendedorismo: uma influência mutuamente vantajosa. Comportamento Organizacional e Gestão, 13(1), 93-114. Recuperado de http://www.scielo.mec. $\mathrm{pt} / \mathrm{pdf} / \mathrm{cog} / \mathrm{v} 13 \mathrm{n} 1 / \mathrm{v} 13 \mathrm{n} 1 \mathrm{a06}$.pdf

Paludo, S. S. \& Koller, S. H. (2007). Psicologia positiva: uma nova abordagem para antigas questões. Paidéia, 17(36), og-20. http://dx.doi.org/10.1590/S0103863X2007000100002

Pereira, M. M., Ferreira, M. C., \& Valentini, F. (2019). Occupational satisfaction of physicians: the impact of demands and resources. Paidéia, 29, 01-09. https:// dx.doi.org/10.1590/1982-4327e2920

Rebolo, F. \& Bueno, B. O. (2014). O bem-estar docente: limites e possibilidades para a felicidade do professor no trabalho. Acta Scientiarum Education, 36(2), 323331. http://dx.doi.org/10.4025/actascieduc.v36i2.21222

Rolando, L. G. R., Salvador, D. F., Vasconcellos, R. F. R. R., \& Luz, M. R. M. P. (2018). Evidências de validade da versão adaptada para o português do questionário TPACK Survey For Meaningful Learning. Avaliação Psicológica, 17(1), 37-47. http://dx.doi.org/10.15689/ ap.2017.1701.05.13157
Salessi, S. \& Omar, A. (2018). Inventario de flow en el trabajo: evidencias de validez de la estructura interna y criterio. Avaliação Psicológica, 17(3), 378-388. https://dx.doi.org/10.15689/ap.2018.1703.14741.11

Schaufeli, W., Dijkstra, P., \& Vazquez, A. C. (2013). Engajamento no trabalho. São Paulo: Casa do Psicólogo

Seligman, M. E. P. (2004). Felicidade Autêntica: usando a nova psicologia positiva para a realização permanente. Rio de Janeiro: Objetiva.

Seligman, M. E. P. (2011). Florescer - uma nova e visionária interpretação da felicidade e do bem-estar. Rio de Janeiro: Objetiva.

Seligman, M. E. P. \& Csikszentmihalyi, M. (2000). Positive psychology: an introduction. American Psychologist, 55(1), 5-14

Seligman, M. E. P. \& Csikszentmihalyi, M. (2014). Positive psychology: an introduction (pp. 279-298). New York: Springer Netherlands.

Seligman, M. E. P. \& Peterson, C. (2003). Foreword: The past and future of positive psychology. In C. L. M. Keyes, \& J. Haidt (Eds.), Flourishing: positive psychology and the life well lived (pp. 11-20). Washington: American Psychological Association.

Seligman, M. E. P. \& Peterson, C. (2003). Positive clinical Psychology. In L. G. Aspinwall, \& U. M. Staudinger (Eds.), A psychology of human strengths: fundamental questions and future directions for a positive psychology (Cap. 21, pp. 305-317). Washington: American Psychological Association

Silva Filho, A. L. A. \& Ferreira, M. C. (2015). O impacto da espiritualidade no trabalho sobre o bem-estar laboral. Psicologia: Ciência e Profissão, 35(4), 1171-1187. https://dx.doi.org/10.1590/1982-3703002482013

Silva, N. \& Boehs, S. D. T. M. (2017). Psicologia positiva: historicidade, episteme, ontologia, natureza humana e método. In S. D. T. M. Boehs, \& N. Silva (Orgs.), Psicologia positiva nas organizações de trabalho: conceitos fundamentais e sentidos aplicados (pp. 2141). Florianópolis: Universidade Federal de Santa Catarina.

Tavares, J. (Org.) (2002). Resiliência e educação. São Paulo: Corte

Vazquez, A. C. S., Magnan, E. S., Pacico, J. C., Hutz, C. S., \& Schaufeli, W. B. (2015). Adaptation and validation of the brazilian version of the Utrecht Work Engagement Scale. Psico-USF, 20(2), 207-217. https:// doi.org/10.1590/1413-82712015200202

Vazquez, A. C. S., Pianezolla, M., \& Hutz, C. S. (2018) Assessment of psychosocial factors at work: a systematic review. Estudos de Psicologia, 35(1), 05-13. https://dx.doi.org/10.1590/1982-02752018000100002

Youssef, C. M. \& Luthans, F. (2007). Positive organizational behavior in the workplace: the impact of hope, optimism, and resilience. Journal of Management, 33(5), 774-800. https://doi. org/10.1177/014.9206307305.562 
Yunes, M. A. M. (2003). Psicologia positiva e resiliência: o foco no individuo e na família. Psicologia em Estudo, 8, 75-84. Recuperado de http://www.scielo $\mathrm{br} / \mathrm{pdf} / \mathrm{pe} / \mathrm{v} 8 \mathrm{nspe} / \mathrm{v} 8$ nesa10.pdf

\section{Endereço para correspondência:}

Silvio José Lemos Vasconcellos

Universidade Federal de Santa Maria

Av. Roraima, 1000, Prédio 74B, sala 3204

Cidade Universitária, Camobi - 97105-900

Santa Maria, RS, Brasil

\section{Adaiane Amélia Baccini}

Mestra em Psicologia com ênfase na Área da Saúde pela Universidade Federal de Santa Maria (UFSM), em Santa Maria, RS, Brasil; psicóloga organizacional do Hospital Universitário de Santa Maria (HUSM), em Santa Maria, RS, Brasil.

\section{Andrea de Fátima Machado da Silva}

Especialista em Docência técnica e profissional pelo sistema de ensino gaúcho (SEG), em Santa Maria, RS, Brasil; psicóloga clinica em consultório privado, em Santa Maria, RS, Brasil.

\section{Carlie da Fontoura Taschetto}

Mestra em Enfermagem pela Universidade Federal de Santa Maria (UFSM), em Santa Maria, RS, Brasil; Oficial Temporária da Força Aérea Brasileira (FAB), em Santa Maria, RS, Brasil.

\section{Jairo Manzoni Rodrigues}

Especialista em Comportamento Organizacional e Gestão de Pessoas pela Escola Superior Aberta do Brasil (ESAB), em Santa Maria, RS, Brasil; psicólogo na Faculdade Integrada de Santa Maria (FISMA), em Santa Maria, RS, Brasil.

\section{Priscila Flores Prates}

Mestra em Psicologia com ênfase na Área da Saúde pela Universidade Federal de Santa Maria (UFSM), em Santa Maria, RS, Brasil; psicóloga clínica, em Santa Maria, RS, Brasil.

\section{Silvio José Lemos Vasconcellos}

Doutor em Psicologia pela Universidade Federal do Rio Grande do Sul (UFRGS), em Porto Alegre, RS, Brasil; professor da Universidade Federal de Santa Maria (UFSM), em Santa Maria, RS, Brasil. 